\title{
Técnicas para facilitar o diagnóstico da topografia da córnea in vivo
}

\author{
Techniques for facilitating in vivocorneal topography diagnosis
}

Luis Alberto Vieira de Carvalho ${ }^{1}$

\footnotetext{
${ }^{1}$ Doutor em Física pelo Instituto de Física da Universidade de São Paulo - USP - São Paulo (SP) e pós-doutorado em Berkeley - University of Califórnia - EUA.

Endereço para correspondência: Av. Dr. Carlos Botelho, 1465 - São Carlos (SP) CEP 13560-250

E-mail: lavcf@ifsc.usp.br

Recebido para publicação em 15.03.2004

Versão revisada recebida em 25.11.2004

Aprovação em 15.12.2004

Nota Editorial: Após concluída a análise do artigo sob sigilo editorial e com a anuência dos Drs. Paulo André Polisuk e Renato Giovedi Filho sobre a divulgação de seus nomes como revisores dele, agradecemos suas participações nesse processo.
}

\begin{tabular}{|l|}
\hline RESUMO \\
\hline Objetivos: Um dos instrumentos mais utilizados atualmente para o diag- \\
nóstico da córnea humana é o videoceratógrafo (VC). Os resultados do VC \\
são impressos na tela do computador na forma de gráficos codificados por \\
cor cujas linhas de contorno delimitam regiões de mesma curvatura. No \\
entanto não há análise criteriosa na literatura dos benefícios de se utilizar \\
diferentes métodos de visualização para estes mapas. Métodos: Instru- \\
mento desenvolvido em nosso laboratório para topografia da córnea foi \\
utilizado nos estudos e para coleta de dados in vivo. A córnea com \\
ceratocone de paciente voluntário foi utilizada como fonte de dados de \\
entrada para todos os métodos de visualização implementados. Resulta- \\
dos: Vários tipos de mapas de visualização foram gerados. Os vários \\
métodos de visualização foram comparados e constatou-se que para cada \\
anomalia existem métodos que salientam os problemas e métodos que \\
"escondem" estes mesmos defeitos. Discussão: Os aspectos relaciona- \\
dos à visualização científica da córnea têm sido de pouca exploração tanto \\
no Brasil quanto na literatura estrangeira. No entanto, com os resultados \\
apresentados neste primeiro trabalho, acreditamos que seja de fundamen- \\
tal importância aumentar não somente o escopo destes estudos, mas \\
também estendê-lo para outros instrumentos médicos. Conclusão: Os \\
resultados obtidos neste trabalho evidenciam a importância de se fazer a \\
escolha correta do método de visualização científica para este tipo de \\
instrumento.
\end{tabular}

Descritores: Topografia de córnea; Ceratocone/diagnóstico; Diagnóstico por computador; Processamento de imagem assistida por computador

\section{INTRODUÇÃOO}

A computação gráfica (CG) tem atraído o interesse de uma crescente comunidade de estudiosos nos últimos $30 \operatorname{anos}^{(1)}$. Nos últimos 10 anos temse notado o surgimento de áreas que se preocupam mais com as aplicações da CG, do que com a CG em si. As ferramentas e técnicas avançadas de computação gráfica permitiram o surgimento de uma nova área de pesquisa e desenvolvimento: a visualização. Existem diversas definições para o termo visualização. Pode-se dizer que é a tradução de dados e informações para um formato gráfico, com intuito de facilitar a interpretação dos mes$\operatorname{mos}^{(2)}$. Uma das conseqüências imediatas disto é o aumento da produtividade, ou seja, o usuário de um sistema que torna disponível as informações de interesse de maneira gráfica poderá, teoricamente, tomar decisões e fazer análises em menos tempo do que por meios convencionais.

Nosso objetivo neste trabalho foi aplicar métodos tradicionais de visua- 
lização científica aos exames tradicionais de um instrumento oftálmico denominado topógrafo de córnea ou, mais formalmente, videoceratógrafo (VC). Como veremos, o VC é um instrumento para medidas da forma da superfície anterior da córnea e é largamente utilizado em clínicas e hospitais para avaliações pré e pós-cirúrgicas de várias naturezas, principalmente em casos de cirurgias de catarata e cirurgias refrativas a laser para correção de ametropias (PRK e LASIK, do inglês, "photorefractive keratectomy" e "laser-in-situ keratomileusis", respectivamente). Como veremos, o método de visualização é bastante importante e se bem utilizado pode levar a um diagnóstico mais preciso.

\section{MÉTODOS}

\section{Topografia da córnea}

A córnea é responsável pela maior parte do poder de refração total do sistema óptico do olho. Há 150 anos pesquisadores têm tentado descrever suas características topográficas $^{(3)}$. O método mais antigo de análise topográfica utiliza as características especulares de reflexão da superfície de lágrima que se forma sobre a porção anterior da córnea. Assim, esta pode ser tratada opticamente como um espelho. Diversos tipos de imagens, em formato de linhas, quadrados, e anéis concêntricos, eram projetadas na superfície anterior para que, através de análises subjetivas, a topografia desta região pudesse ser investigada.

Em 1880, o português A. Plácido ${ }^{(4)}$ desenvolveu um sistema de projeção que está em uso até os dias de hoje. O projetor era um disco com anéis brancos e pretos alternados, com um furo no centro por onde o observador poderia visualizar a córnea do paciente (Figura 1A). Este sistema melhorou bastante o processo de alinhamento, pois bastava o paciente fixar a visão ao centro do projetor para que este estivesse alinhado ao eixo de visão. Se o padrão refletido fosse o de anéis aproximadamente circulares e concêntricos, a córnea poderia ser interpretada como aproximadamente esférica (Figura 1B); do contrário, a topografia seria de uma superfície irregular (Figura 1C).

Com o barateamento e compactação de sistemas computa-

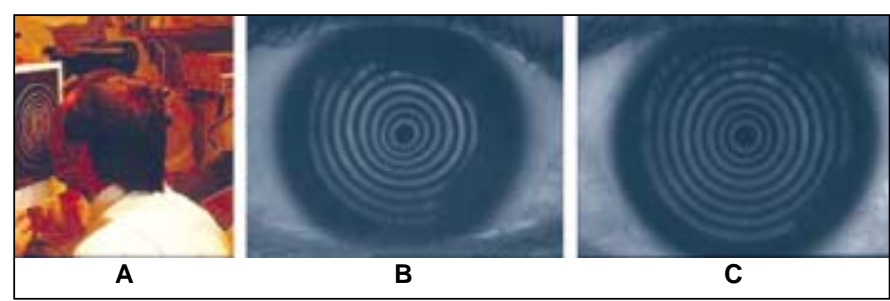

Figura 1 - (A) Foto que demonstra o princípio dos anéis de Plácido ${ }^{(4)}$ (B) O padrão refletido pela córnea do paciente pode indicar anomalias na topografia da córnea. A simetria e concentricidade dos anéis refletidos são características típicas de uma córnea aproximadamente esférica; (C) Distorção dos anéis refletidos indicam ser esta uma córnea com uma topografia irregular cionais a partir da década de 80 , a possibilidade de análises da topografia da córnea com auxílio de instrumentos computadorizados começou a tornar-se uma realidade. Uma série de benefícios foi implementada. As medidas são realizadas com maior rapidez e dependiam cada vez menos da habilidade do oftalmologista. As imagens eram processadas computacionalmente em um tempo muito menor do que nos fotoceratoscópios. A disposição da curvatura em milhares de pontos sobre a córnea podia ser feita na tela do computador, facilitando o diagnóstico. Além da "explosão" computacional, outros fatores favoreciam o desenvolvimento de instrumentos dessa natureza. O crescente desenvolvimento de técnicas de cirurgias refrativas necessitava de sistemas que pudessem avaliar o sucesso das cirurgias, com análises precisas pré e pós-cirúrgicas. A estes novos instrumentos computadorizados deu-se o nome de topógrafos de córnea ou mais formalmente videoceratógrafos (VC). Na figura 2 temos um diagrama do princípio do topógrafo de córnea.

O sistema de projeção de anéis é geralmente cônico. O cone é normalmente feito em acrílico translúcido com anéis circulares concêntricos pintados em preto por onde não se deseja que a luz passe. A iluminação é feita por uma lâmpada em forma de "biscoito" acoplada na parte traseira do cone. Por trás do projetor, ao longo do eixo deste, existe um sistema óptico de magnificação das imagens refletidas pela córnea (Figura 2). Tais imagens são focalizadas numa câmara CCD ("charege coupling device") monocromática acoplada ao sistema óptico e também alinhada com o eixo deste. O sinal do CCD é enviado a uma placa de aquisição ("frame grabber") instalada no micro-computador. Desta maneira imagens extremamente nítidas e com alta magnificação podem ser digitalizadas (Figuras 1B e 1C). Após a digitalização, as imagens pas-

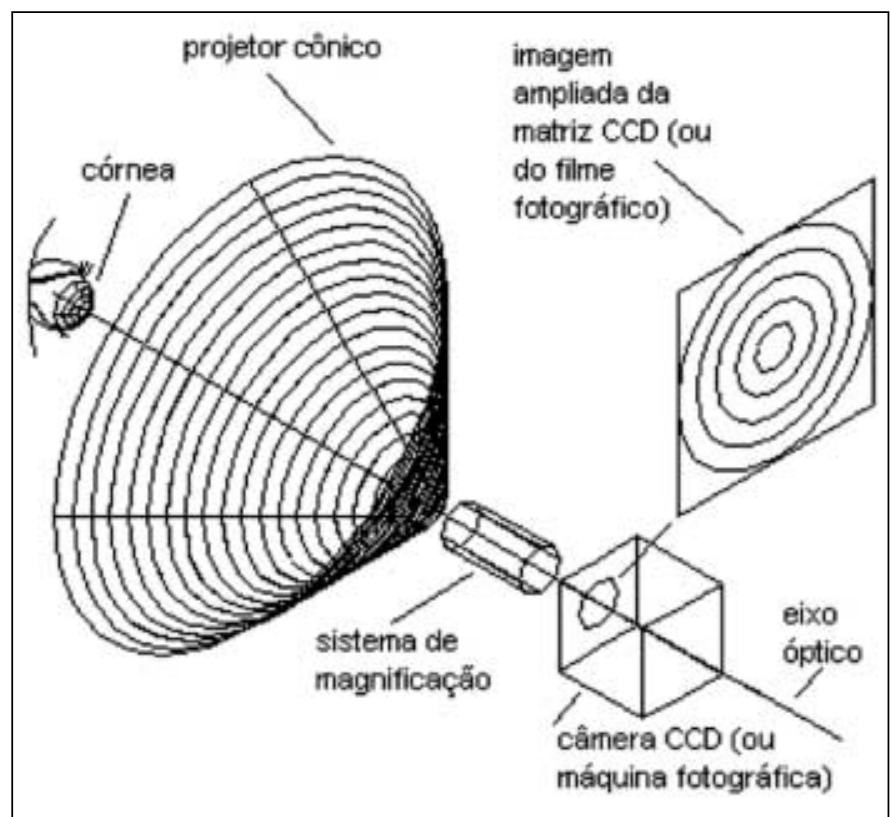

Figura 2 - Princípio de funcionamento do VC 
sam por processamentos computacionais para detecção das bordas internas e externas dos discos. Os valores obtidos nesta etapa são inseridos em algoritmos de reconstrução da topografia da córnea ${ }^{(5-9)}$, cujo resultado é geralmente impresso na tela do computador em forma de mapas topográficos com códigos de cores (Figura 3). Cada cor da escala representa uma curvatura determinada, ou seja, um certo poder de refração (dioptria). Cores quentes (vermelho, laranja e amarelo) representam altos graus de curvatura. Os tons em verde representam valores médios enquanto cores frias (azul, azul escuro) estão associadas a curvaturas menores.

\section{Visualização científica}

Na visualização científica deseja-se explorar dados numéricos e outras informações, provindas de áreas científicas (Engenharia, Biologia, Física, etc.) e dispô-los de tal maneira a facilitar ou melhorar sua compreensão e análise. "O propósito da computação é inspiração, e não números"(1). Neste trabalho estaremos dando ênfase a este ramo da Visualização, com a aplicação específica numa área da medicina - a oftalmologia.

\subsection{Tipos de dados}

Denota-se por $E_{n}^{S}$ uma entidade escalar $S$ definida sobre um domínio de dimensão $n$. Alguns exemplos são: $E_{2}^{V_{2}}$ denota um conjunto de vetores bidimensionais definidos sobre um domínio 2D; um conjunto de pontos esparsos definido sobre um domínio 3D é denotado por $E_{3}^{P}$. A entidade pode ser definida ponto a ponto ou sobre regiões em um domínio contínuo, ou um conjunto enumerado. A notação $E_{[n]}$ indica um domínio ndimensional com valores definidos sobre regiões; $E_{\{n}$ indica um domínio n-dimensional que consiste de valores enumera- dos. Existem técnicas específicas para cada tipo de conjunto de pontos: enumerados, escalares, vetoriais e tensoriais.

A tabela 1 mostra técnicas de visualização para entidades escalares e vetoriais $^{(2)}$. Mais adiante, nos resultados, apresentamos exemplos de cada uma destas técnicas aplicadas à córnea.

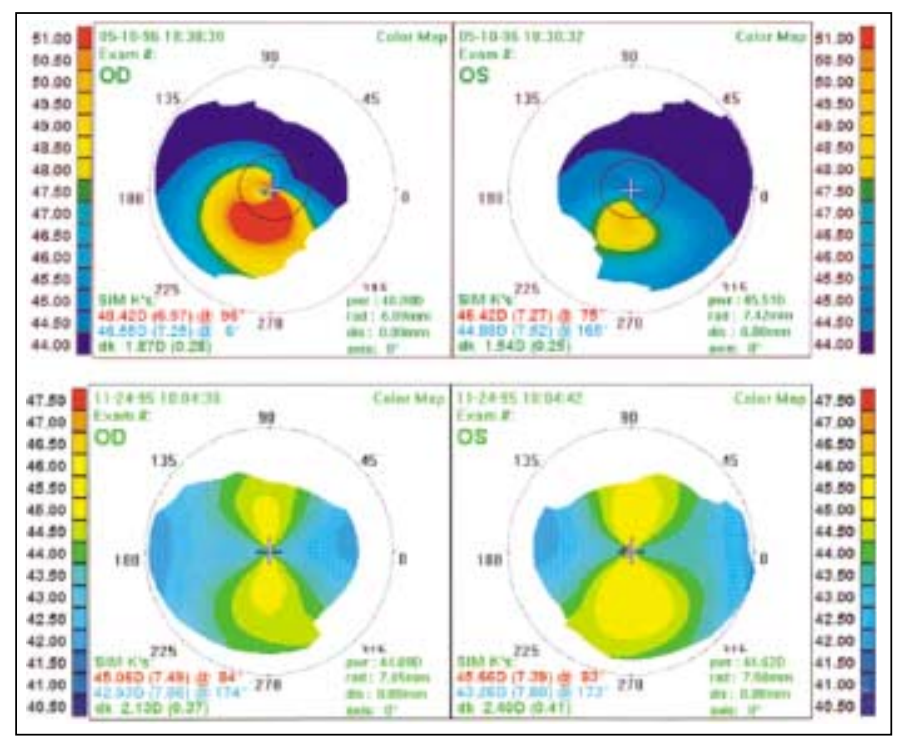

Figura 3 - Exemplo das telas de mapas topográficos de um equipamento comercial. Geralmente estes mapas são praticamente os únicos dados que oftalmologista tem para poder fazer uma série de diagnósticos da córnea. Valores no canto inferior direito de cada mapa indicam, de baixo para cima e com relação à posição do cursor na tela do computador: dioptria, raio de curvatura, distância ao centro, eixo. Valores no lado oposto referem-se ao eixo e grau de astigmatismo. Os mapas de cima são de um paciente com ceratocone (altas curvaturas localizadas). Os mapas abaixo evidenciam um caso típico de astigmatismo a favor da regra.

\begin{tabular}{|c|c|c|c|}
\hline \multirow[b]{3}{*}{ Dimensão do domínio } & \multicolumn{2}{|c|}{ Tipos de dados representáveis } & \\
\hline & \multirow[b]{2}{*}{ Escalar } & \multicolumn{2}{|c|}{ Vetorial } \\
\hline & & 2D & 3D \\
\hline 1 & Gráfico de linhas & & \\
\hline [1] & Histograma & & \\
\hline$\{1\}$ & Gráfico de Barras & & \\
\hline 2 & $\begin{array}{l}\text { Mapeamento sobre superfícies } \\
\text { Height fields } \\
\text { Mapeamento por cores, imagens, isolinhas }\end{array}$ & $\begin{array}{l}\text { Ícones 2D } \\
\text { Linhas de escoamento }\end{array}$ & $\begin{array}{l}\text { Ícones 3D em planos de corte } \\
\text { Geração de escalares } \\
\text { Mapeamento por cores }\end{array}$ \\
\hline [2] & $\begin{array}{l}\text { Traçado de regiões } \\
\text { Histograma } 2 D \\
\text { Gráfico de barra } 2 D\end{array}$ & & \\
\hline 3 & $\begin{array}{l}\text { Isosuperfícies } \\
\text { Renderização volumétrica direta } \\
\text { Mapeamento por cores }\end{array}$ & & $\begin{array}{l}\text { Ícones 3D } \\
\text { Geração de escalares } \\
\text { Linhas de escoamento 3D } \\
\text { Superfícies de escoamento } \\
\text { Fitas de escoamento } \\
\text { Texturas sobre superfícies } \\
\text { Animação }\end{array}$ \\
\hline $3+$ tempo & & Animação & \\
\hline
\end{tabular}




\section{Visualização científica aplicada à córnea}

Como vimos até agora, os exames topográficos são geralmente dispostos na forma de mapas coloridos bidimensionais (Mapeamento por cores - veja figura 3 - "Color mapping"), onde cada cor está associada a um poder dióptrico e as posições de cada cor no plano bidimensional representam posições físicas da córnea na imagem dos discos de Plácido digitalizada no VC. Seria esta a maneira mais atraente para o oftalmologista analisar os dados de interesse? Será que esta é a maneira mais conveniente de visualização da córnea dentro do contexto que o oftalmologista está habituado a trabalhar?

As respostas a estas questões não são muito simples. Numa primeira aproximação, todas as córneas têm uma forma aproximadamente esférica. Quando analisada com maior precisão, percebe-se que são as pequenas variações que distinguem uma córnea da outra. São estas pequenas variações que podem causar diferenças na acuidade visual do paciente ${ }^{(10)}$. As informações somente da curvatura (ou de uma grandeza proporcional à curvatura) em função da posição não são suficientes para que o oftalmologista possa tomar todas as decisões com relação aos procedimentos que devem ser seguidos.

Existem vários métodos para se computar diferentes grandezas, como curvatura, relacionadas à córnea com base nas imagens dos discos de Plácido ${ }^{(11-14)}$. Não é o escopo deste trabalho descrever em detalhes como são realizados estes cálculos; para tal recomendamos o leitor à referência ${ }^{(13)}$. No entanto, é interessante descrevermos aqui quais as outras grandezas de interesse para a visualização e que têm sido pouco explorada pelos fabricantes de $\mathrm{VC}$ mundo afora.

Com auxílio da figura 4 podemos entender quais os diferentes parâmetros de interesse para um exame completo da superfície corneana. Para determinarmos, por exemplo, a curvatura axial (A - Figura 4) interpola-se cada ponto da superfície da córnea pela melhor esfera com centro sobre o eixo óptico; já para determinar a curvatura tangencial (B - Figura 4) mede-se a curvatura tangencial (ou instantânea) naquele ponto (veja definição precisa de curvatura instantânea na referência ${ }^{(12)}$ ), logo o raio de curvatura não necessariamente está centrado sobre o eixo óptico; para determinar a curvatura ou poder refrativo, que procura simular na córnea a refração dos raios de luz usando a lei de Snell (C - Figura 4), é necessário computar o inverso da distância focal de cada ponto da córnea. Podemos ver na figura 4 (D, E, F) que o modelo de elevação (topografia de córnea propriamente dita) mede as elevações reais de cada ponto da superfície, e pode ser relativo a três superfícies: um plano (D), uma esfera maior que a córnea (E) e uma esfera menor que a córnea (F). Cada um destes parâmetros exige cálculos e algoritmos diferentes, que foram implementados em trabalhos anteriores ${ }^{(8-9)}$.

As técnicas de visualização, quando aplicadas a estes diferentes parâmetros, devem ter como objetivo principal o auxílio aos seus principais potenciais usuários, ou seja, os oftalmologistas. Deve-se levar em consideração os hábitos desta audiência, ou seja, como eles estão acostumados a interpretar informações relativas à córnea. Um sistema que exigisse maneiras completamente novas de análise certamente iria ser desprezado pela comunidade oftalmológica. Com base nisso, implementamos neste trabalho técnicas de visualização usando alguns dos métodos descritos na tabela 1 para parâmetros calculados com base na figura 4. Para tal, foram utilizadas interfaces gráficas o mais próximo possível daquilo que o médico oftalmologista está acostumado nos "softwares" comerciais (Figura 3). A seguir mostramos os resultados obtidos usando várias técnicas e discutimos as vantagens de cada um ao final do trabalho.

\section{RESULTADOS}

\section{Tipos de dados}

Como mencionado na figura 4, existem diversos parâmetros que podem ser calculados a partir dos dados coletados pelas imagens de Plácido. No entanto, pelos conceitos introduzidos na seção anterior podemos afirmar que, embora iremos utilizar diversos métodos de visualização diferentes, nossos dados são classificados como $E_{[2}^{S}$, ou seja, um conjunto de dados escalares definido sobre regiões em um domínio bidimensional.

Para expor os resultados para estes dados, utilizando as diferentes técnicas de visualização, de maneira didática e ao mesmo tempo o mais completa possível, resolvemos dividir as subseções a seguir de acordo com a seqüência de parâmetros mostrados na figura 4. Primeiramente vamos mostrar os resultados obtidos para o cálculo das curvaturas axiais e todas as variações e técnicas aplicadas a estes dados; em segundo, vamos aplicar as mesmas técnicas mas desta vez para dados da curvatura tangencial; depois vamos mostrar os resultados para mapas de curvatura (ou poder dióptrico) refrativos. Fi-

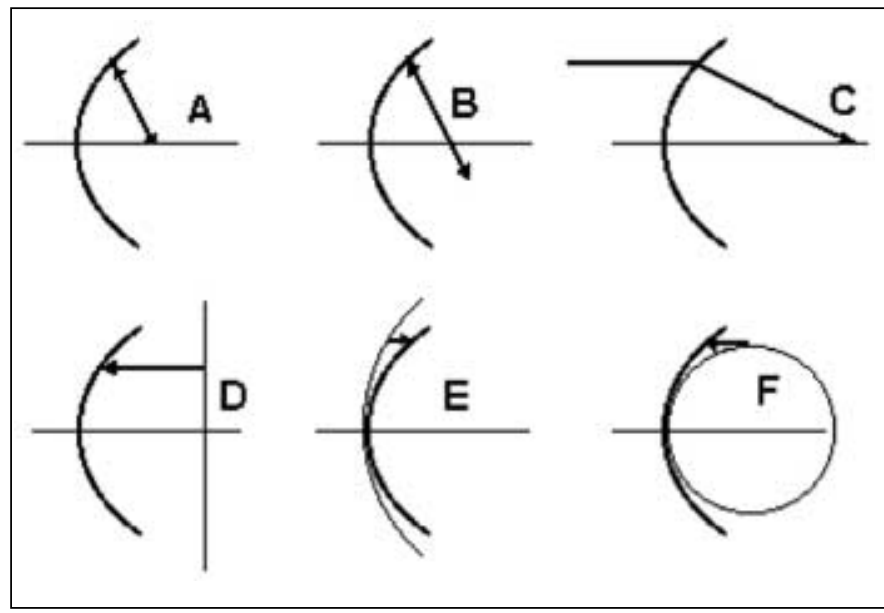

Figura 4 - Os diferentes modelos para descrição da superfície da córnea. Cada um tem vantagens e desvantagens entre si no momento de se avaliar a qualidade óptica da córnea. Uma análise detalhada destes fatores foi feita por Salmon ${ }^{(13)}$ e Klein ${ }^{(14)}$ 
nalmente, iremos expor os resultados para os dados da elevação relativa da córnea e mostraremos que, como estes dados são muito sutis (pouquíssima variação de um ponto para outro sobre a superfície) devem-se aplicar técnicas bastante específicas para facilitar a visualização destas diferenças.

Com isso, além de apresentarmos métodos diferentes de visualização das grandezas com as quais os oftalmologistas já estão acostumados, apresentamos também métodos aplicados a grandezas (parâmetros) que até então eram pouco ou quase nunca utilizadas nas clínicas e hospitais.

\section{Poder dióptrico (ou simplesmente Curvatura) versus Posição (dimensão do domínio [2], mapeamento por cores e isolinhas)}

\section{Curvatura axial (A - Figura 4)}

Como vimos na seção 2.1, este é um dos mapas mais utilizados em topografia de córnea atualmente. A maioria dos aparelhos comerciais traz limitações que podem ser superadas com algumas modificações. Isto se deve principalmente ao fato do médico oftalmologista estar habituado a parâmetros como curvatura e poder de refração.

Antes de calcular o poder refrativo axial é necessário calcular o raio de curvatura axial. Como vimos na figura 4 , este raio pode ser definido como a distância do ponto considerado sobre a superfície da córnea até o eixo axial, sendo que estes dois pontos estão localizados sobre o segmento de reta normal à córnea no ponto considerado. Para calcular este raio de curvatura baseado nos discos de Plácido utilizamos um algoritmo complexo. Como uma explicação detalhada deste algoritmo não é do escopo deste recomendamos o leitor interessado a trabalhos anteriores ${ }^{(5-9)}$.

Como o conceito de poder dióptrico, embora diretamente proporcional à curvatura, é um tanto incomum em computação gráfica ou mesmo em outros ramos da visualização, descrevemos a seguir, de maneira resumida, o conceito de dioptria, amplamente utilizado em oftalmologia. O poder de refração de uma lente, ou seja, o quanto ela é capaz de convergir os raios de luz incidentes, é inversamente proporcional ao raio de curvatura da superfície e diretamente proporcional ao seu índice de refração. Para a córnea, cuja função no olho é exatamente a de uma lente, não é diferente. Utilizando-se notação matricial pode-se deduzir ${ }^{(5-6)}$ uma equação para a dioptria de cada ponto sobre a córnea:

$$
D=\frac{n_{c}-n_{a r}}{r}=\frac{1}{f}
$$

onde: D: dioptria; $n_{c}$ : índice de refração da córnea $=1,3375$; $n_{a r}$ : índice de refração do ar $=1,0000 ; r$ : raio de curvatura.

A restrição da maioria dos aparelhos comerciais é a falta de possibilidade e flexibilidade na variação das escalas dióptricas, ou do intervalo da escala, apesar de ser algo simples de se implementar via "software". Na figura 5 implementamos mapas do nosso paciente com ceratocone com várias escalas diferentes para demonstrar a importância na escolha da escala.
Analisando a figura 5 podemos inferir alguns detalhes importantes: (A) Mapa usando escala com intervalo de 0,5 dioptrias. Podemos perceber nitidamente a região de maiores curvaturas e a escala se distribui por inteiro ao longo de toda a superfície da córnea, portanto facilitando a visualização das anomalias no poder dióptrico; (B) Mesma córnea mas com escala com intervalo de 2D. Podemos perceber que o ceratocone, que é uma região anômala, não está aparecendo de maneira destacada, ou seja, aos olhos desatentos, poder-se-ia pensar que se trata até mesmo de um astigmatismo irregular, algo muito menos grave do que um ceratocone e que poderia levar a procedimentos errôneos na sua correção; (C) Mapa com escala absoluta de cores. Neste tipo de mapa todas as dioptrias são associadas a valores constantes de cor e podemos perceber que as regiões anômalas são destacadas satisfatoriamente; a única vantagem deste tipo de mapa com relação ao mostrado em (A) é que mapas deste tipo podem ser comparados com outros mapas, mesmo para córneas de pacientes diferentes, pois a escala é sempre a mesma; (D) Mapa limiar ou "Threshold", do inglês. Este mapa imprime as curvaturas em apenas duas cores, sendo que em azul aquelas abaixo de um certo valor e em vermelho aquelas acima deste mesmo valor. A vantagem principal deste mapa é que ele permite uma interação direta com o usuário, que pode mudar o valor do limiar e analisar o mapa para identificar regiões acima e abaixo daquele valor de curvatura. Conforme podemos perceber pela figura o ceratocone fica bem delimitado para valor de limiar de 55,5D.

\section{Curvatura tangencial (B - Figura 4)}

A curvatura tangencial é proporcional ao inverso do raio de curvatura instantânea, e não está restrito a ter sua origem posicionada sobre o eixo óptico (ou eixo axial) como é o caso da curvatura axial (A). Não iremos reproduzir aqui, por motivos de espaço e também por não ser o escopo deste manuscrito, mas podemos afirmar que o cálculo da curvatura instantânea pode ser realizado utilizando-se o cálculo diferencial inte-

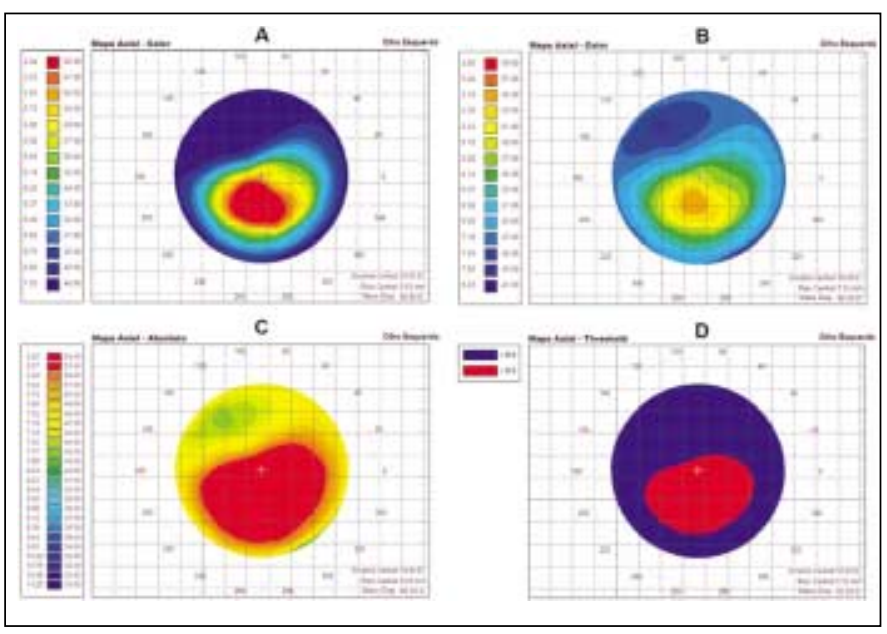

Figura 5 - Mapa do tipo Axial dos poderes dióptricos do ceratocone com várias escalas diferentes 
gral. Imaginem que a intersecção de um plano, que contém o eixo óptico visual, com a superfície da córnea possa ser descrita por uma equação $f(x)$. Do cálculo diferencial-integral sabese que a curvatura em cada ponto desta função pode ser expressa como ${ }^{(12)}$ :

$$
k(x)=\frac{f^{\prime \prime}(x)}{\left[1+\left(f^{\prime}(x)\right)^{2}\right]^{3 / 2}}
$$

e sabe-se que o raio de curvatura é o inverso da curvatura

$$
r=\frac{1}{k(x)}
$$

Utilizando-se a mesma equação (1) para o cálculo do poder dióptrico, basta multiplicar $k(x)$ por $\left(n_{c}-n_{a r}\right)$. Assim são calculados milhares de valores de poder dióptrico tangencial para a mesma córnea com ceratocone utilizada em todos exemplos apresentados neste trabalho. Na figura 6 podemos ver os mesmo tipos de mapas da figura 5 mas desta vez para o poder dióptrico tangencial:

A análise destes gráficos é praticamente idêntica àquela para a curvatura axial. Apenas há uma diferença intrínseca aos valores de curvatura pois no modelo tangencial as regiões de altas curvaturas ficam um pouco mais localizadas (região do ceratocone fica um pouco menor).

\section{Curvatura refrativa (C - Figura 4)}

Conforme vimos na figura 4 quando utilizamos os dados da topografia de córnea em conjunto com a lei de Snell para refração, podemos traçar raios do infinito entrando no olho. A distância do ápice da córnea até a intersecção dos raios refratados com o eixo óptico, caracteriza a distância focal $(f)$ daquele feixe. Como vimos pela equação (1), o inverso da distância focal também caracteriza o poder dióptrico de uma lente. É desta maneira que os valores de poder dióptrico refrativo foram calculados. Vamos aplicar as mesmas técnicas de visualização utilizadas para o poder axial e tangencial anteriores.

Para a curvatura refrativa notamos uma diferença mais acentuada quando comparada à curvatura axial e tangencial, principalmente com relação aos valores máximo e mínimo da escala. Neste sentido o mapa absoluto (C) mostra-se bastante importante pois somente ele permite a comparação direta com os mapas axial e tangencial. Conforme podemos perceber pelos três mapas absolutos (Figura 5-C, Figura 6-C e Figura 7-C) as dioptrias refrativas abrangem uma escala maior de valores. Mas isso era esperado pois conforme a figura 4 podemos ver que se trata de um modelo bastante diferente dos outros. Mesmo assim, percebemos que a escala que melhor serve para identificar o ceratocone é aquela com intervalo de $0,5 \mathrm{D}$. No entanto, na figura 7-D escolhemos um valor de limiar para mostrar que, caso este parâmetro não seja bem escolhido, este mapa de pouco adianta para salientar alguma região pesquisada e toda superfície ficará com apenas uma tonalidade. Para os outros mapas (A, B) podemos perceber que ficaram bastante parecidos com seus equivalentes tangencial e axial, deixando claro o conceito de escala relativa, que é um tipo de escala que se adapta a cada mapa impresso.

\section{Visualização tridimensional do poder dióptrico axial, tangencial e refrativo (domínio 2D, isosuperfícies, mapea- mento por cores)}

Implementamos dois métodos para exposição de mapas 3D da córnea: (1) supondo-se um sistema cartesiano no espaço (eixos $\mathrm{x}, \mathrm{y}, \mathrm{z}$ ) pode-se imprimir os valores de dioptria num eixo (geralmente o z) e de posição em mm nos outros eixos; (2) imprimimos o valor da elevação propriamente dita da córnea em mm (eixo z) versus posição em mm (eixos x e y). No caso (1) utilizam-se os dados dióptricos pontuais, calcula-se valores de dioptria para milhares de pontos sobre a córnea e imprimem-se estes valores no eixo $z$, e a posição nos outros eixos. $\mathrm{Na}$ figura 7 podemos ver dois exemplos destes mapas para nosso paciente com ceratocone.

Resolvemos imprimir todos estes valores em uma mesma figura para que a comparação dos valores fique mais fácil, já que, pelo menos a curvatura tangencial e axial são bastante próximas. Na figura 8 podemos ver os três mapas de curvatura

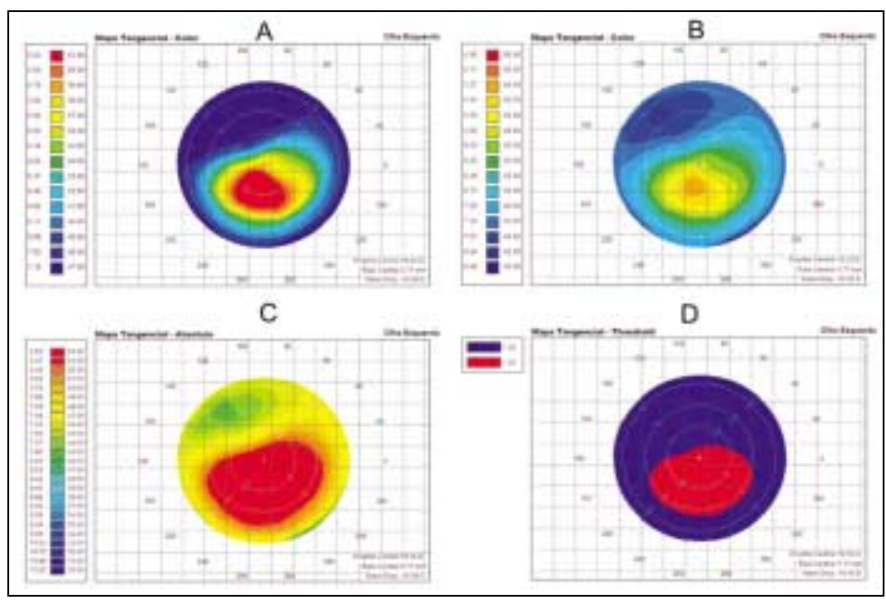

Figura 6 - Mapas planos coloridos da curvatura tangencial com escalas diferentes

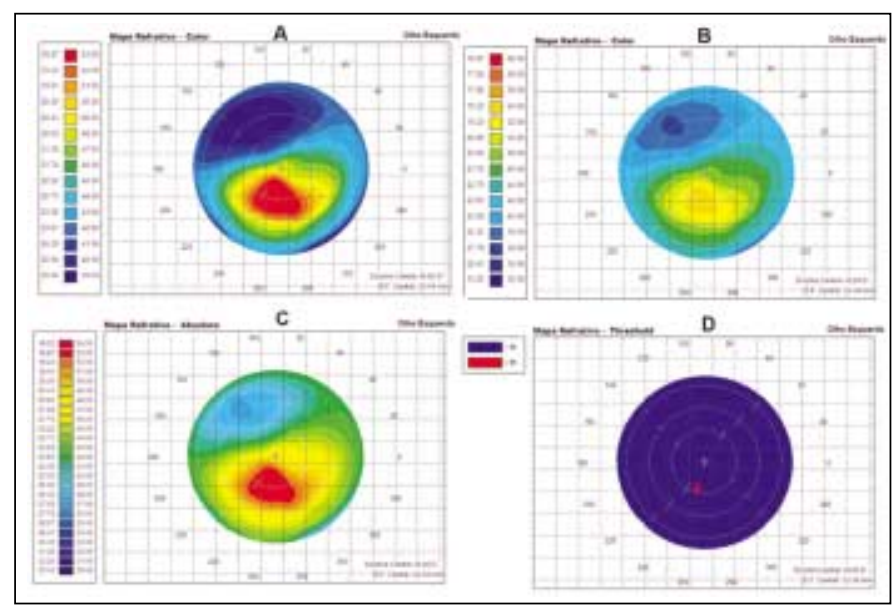

Figura 7 - Mapas planos coloridos das curvaturas refrativas. Podemos perceber uma grande diferença nos valores das pontuais com relação aos mapas axial e tangencial 


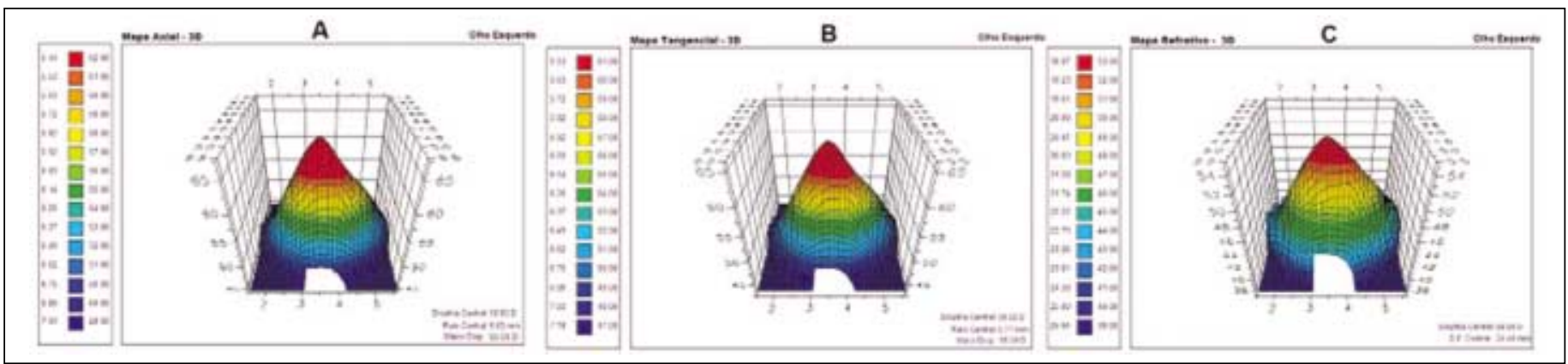

Figura 8 - Mapas tridimensionais da curvatura versus posição: (A) Axial, (B) Tangencial e (C) Refrativa. Embora todos os mapas pareçam idênticos há uma diferença na escala, principalmente para o mapa refrativo. Como podemos perceber os mapas tridimensionais são difíceis de analisar e podem induzir a erros. Uma maneira prática de deixar estes mapas mais didáticos é gerar códigos que permitam ao usuário interagir com o mapa, mudando sua escala, fazendo zoom e mudando o ângulo da perspectiva

codificados por cor, mas desta vez impressos como superfícies 3D onde no eixo $x$, $y$ temos a posição física propriamente dita da córnea e no eixo $\mathrm{Z}$ temos os valores de curvatura. Muitos médicos costumam confundir estes gráficos com mapas tridimensionais da elevação propriamente dita. Como veremos mais adiante não há relação nenhuma entre os mapas 3D de curvatura e 3D de elevação física da córnea.

\section{Elevação (D, E e F) (dimensão do domínio [2], mapeamento por cores e isolinhas, gráficos tridimensionais, cortes transversais)}

Iremos tratar aqui dos mesmos tipos de visualização empregados anteriormente para curvatura tangencial, axial e refrativa, mas com a diferença de que desta vez nossos valores escalares são as altitudes (ou elevações) propriamente ditas da córnea (D, E, F - Figura 4). Para estes dados, assim como em mapas topográficos usados em geografia, imprimem-se mapas coloridos planos onde a cor está associada a uma tabela de altitudes. Sabe-se há muito tempo ${ }^{(15)}$ que a maior parte das córneas tem formato bastante próximo ao de esferas. Seria então interessante a subtração de superfícies esféricas do formato original para que o usuário tenha uma percepção mais apurada de quanto e onde a córnea difere de uma superfície perfeitamente esférica (veja E, F - Figura 4). O resultado desta subtração para esferas de diferentes raios de curvatura pode ser visto nas figuras 5B, 5C e 5D.

Embora pareça um método interessante, surgem vários problemas. Primeiro, não fica muito claro como definir a melhor superfície esférica para subtração. Pode-se, por exemplo, definir uma superfície cujo volume esteja completamente dentro da superfície da córnea. Pode-se também definir uma superfície que interseciona a córnea. Enfim, não fica muito claro qual seria a melhor definição para esta superfície.

Apesar de ser uma maneira bastante didática de representar superfícies, acaba tendo falhas no momento de evidenciar pequenas variações na superfície, devido aos intervalos da tabela. Além disso, a transformação mental do mapa plano para uma superfície tridimensional pode não ser tão simples para alguns usuários.

\section{DISCUSSÃO}

Um dos detalhes mais importantes, quando comparamos as mesmas córneas depois de visualizadas de várias maneiras, é a escala. Embora alguns tipos de visualização chamem atenção para certos detalhes da córnea, é fundamental que a escala seja sempre impressa junto ao mapa e que, mais do que isso, o médico observe atentamente seus valores limites (inferior e superior). É este uma das maiores fontes de erro de médicos que estão iniciando a residência em oftalmologia. No caso específico dos mapas 3D para tangencial, axial e refrativo, é interessante notar que, embora os valores dióptricos sejam bastante diferentes, todos os gráficos da figura 8 parecem muito entre si. Isto nos leva a pensar em mais um tipo de mapa, que poderia ser implementado numa segunda fase do trabalho, para solucionar este problema. Este mapa seria a impressão da curvatura tridimensional com escala absoluta. Desta maneira poderíamos salientar com maior facilidade as diferenças, pois tanto as cores como as elevações seriam diferentes.

Com relação aos mapas de elevação relativa às esferas menor e maior que a córnea, fica evidente que são muito mais sensíveis do que os mapas relativos ao plano (Figura 9). Isto tem um motivo evidente, pois as variações de distância da superfície com relação ao plano são muito menores do que com relação às esferas. No entanto, deve-se salientar que uma melhoria deste método seria o cálculo da diferença com relação a uma esfera ou toróide que mais se aproximasse da superfície da córnea. Neste sentido, análises ainda mais refinadas das diferenças poderiam ser feitas.

Outro aspecto que pudemos perceber ao longo das pesquisas é que todo sistema de visualização em saúde deve considerar aquilo que o profissional já está acostumado, ou aquilo que ele aprendeu ao longo de anos de trabalho e diagnóstico. Devem-se introduzir soluções que não exigem uma mudança completa de filosofia ou um aprendizado completamente novo a respeito de algum sistema. Isto vale também para a interface gráfica do sistema (GUI).

A próxima etapa deste trabalho será realizada no Departamento de Oftalmologia da Escola Paulista de Medicina (EPM) 


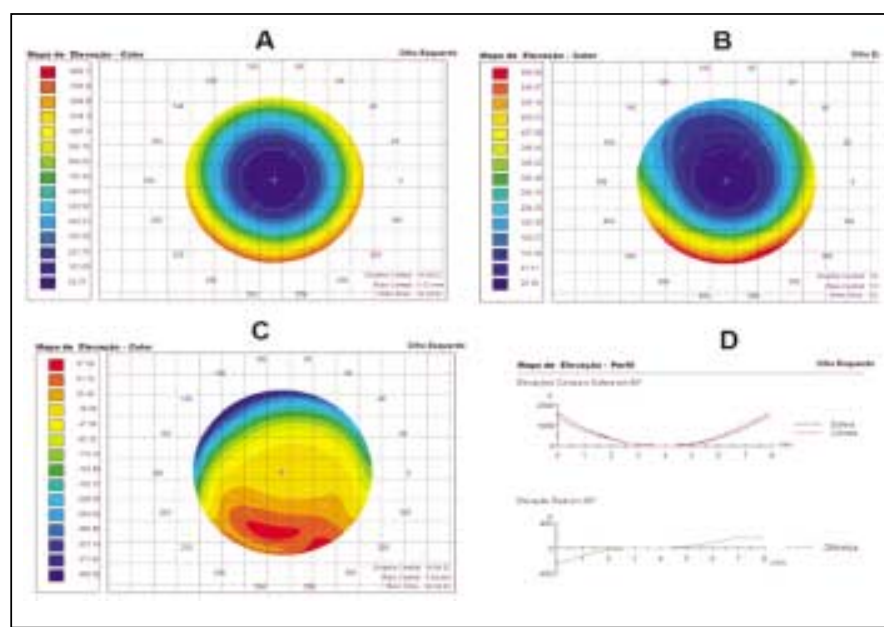

Figura 9 - (A) Elevação relativa ao plano; (B) Elevação relativa a uma esfera menor que a córnea $(5,5 \mathrm{~mm}$ de raio); (C) elevação relativa a uma esfera maior que a córnea $(8,0 \mathrm{~mm}$ de raio) e (D) mapa do tipo "corte transversal" relativo ao mapa em (C), mostrando elevação da córnea (vermelho), da esfera (azul) e a diferença (verde). Podemos perceber que este corte facilita bastante a visualização das diferenças entre a córnea e a esfera para um determinado meridiano

da Universidade Federal de São Paulo (UNIFESP) e na Escola de Medicina de Ribeirão Preto (EMRP) da Universidade de São Paulo (USP), para averiguar clinicamente a utilidade de cada um destes métodos de visualização.

\section{ABSTRACT}

Purpose: The videokeratoscope is one of the most widely used instruments for the diagnosis of the human cornea. Results are usually plotted in the form of color-coded maps where each color is associated with a specific range of curvatures or elevations. Nevertheless there has been no thorough study in the literature demonstrating the advantages or disadvantages of different visualization techniques applied to the videokeratoscope. Methods: A videokeratograph developed in our laboratory was used to collect in vivo data. The cornea of a patient with keratoconus was used as input data for all implemented visualization methods. Results: The various methods of visualization were compared between each other for the same cornea and it was observed that for each method the corneal anomalies appear differently. Discussion: The aspects related to the scientific visualization of the cornea have been a subject of very little attention both by the Brazilian and international community. Nevertheless, with the results presented in this first study, we believe it is not only important to extend the scope of these studies but also to apply these techniques to other medical areas. Conclusion: A simple choice of the visualization method can make the difference between a simple and correct diagnosis and a completely dramatic error in an apparently simple case. Therefore, the results obtained in this study make clear the importance of making the correct choice of the scientific visualization method for this kind of medical instrument.

Keywords: Corneal topography, Keratoconus/diagnosis; Diagnosis, computer-assisted; Image processing, computer-assisted

\section{REFERÊNCIAS}

1. Earnshaw RA, Watson D. Animation and scientific visualization, tools and applications. New York: Academic Press; 1993.

2. Oliveira MCF de, Minghim R. Uma introdução à visualização computacional. In: XVI JAI - Jornada de Atualização em Informática, Brasília-DF, 2-8 Agosto de 1997; p.85-127.

3. Schanzlin DJ, Robin JB. Corneal topography-measuring and modifying the cornea. New York: Springer-Verlag; 1991.

4. Placido A. Novo instrumento de exploração da córnea. Periodico d'Oftalmológica Practica (Lisboa) 1880;(5):27-30.

5. Carvalho LAV, Faria SJS, Castro JC. Desenvolvimento de um sistema para medida da esfericidade da córnea durante a cirurgia. Rev Física \& Instrumentação. 1996;12(3):81-90.

6. Carvalho LAV, Tonissi AS, Romão AC, Santos LE, Yasuoka F, Oliveira AC, et al. Desenvolvimento de um instrumento computadorizado para medida do poder refrativo da córnea (Videoceratógrafo). Arq Bras Oftalmol. 1998;61(6): 640-54.

7. Carvalho L, Tonissi AS, Castro JC. Preliminary tests and construction of a computerized quantitative surgical keratometer. J Cataract Refract Surg. 1999; 25(6):821-6. Comment in: J Cataract Refract Surg. 2000;26(8):1104-5.

8. Carvalho LA, Stefani M, Romão AC, Carvalho L, Castro JC de, Tonissi S, et al. Videokeratoscopes for dioptric power measurement during surgery. J Cataract Refract Surg. 2002;28(11):2006-16.

9. Carvalho LAV de, Romão AC, Tonissi S, Castro FJC, Schor P, Wallace C. Videokeratography (VKS) for monitoring corneal curvature during surgery. Arq Bras Oftalmol. 2002;65(1):37-41.

10. Carvalho LA. Computer algorithm for simulation of the human optical system and contribution of the cornea to the optical imperfections of the eye. Rev Física \& Instrumentação. 2003;16(1):7-17.

11. Carvalho LA, Romão AC. Instrumentação para digitalização e processamento computacional de imagens da pupila in vivo. Rev Física \& Instrumentação. 2003;16(1):24-9.

12. George B, Thomas Jr Ross LF. Calculus and analytic geometry. $9^{\text {th }}$ ed. Massachusets: Addison-Wesley; 1996.

13. Salmon TO, Horner DG. Comparison of elevation, curvature, and power descriptors for corneal topographic mapping. Optom Vis Sci. 1995;72(11):800-8.

14. Klein SA, Mandell RB. Shape and refractive powers in corneal topography. Invest Ophthalmol Vis Sci. 1995;36(10):2096-109.

15. Mandell RB, York MA. A new calibration system for photokeratoscopy. Am J Optom Arch Am Acad Optom. 1969;46(6):410-7.

Nos artigos enviados para publicação, o nome dos autores e suas afiliações devem estar completos. Isso facilitará a indexação e os links com as bases de dados e o CV Lates. 